\title{
ESPECTROS ÓPTICOS DE ABSORÇÃO E DADOS COLORIMÉTRICOS DE GRANADAS GEMOLÓGICAS DA BACIA DO RIO SANTO INÁCIO, MUNICÍPIO DE COROMANDEL-MG.
}

\author{
WOLDEMARIWANUCH* , STANISLAV STEPANOVICH MATSYUK** \& MIKHAIL NIKOLAEVICH TARAN**
}

\begin{abstract}
OPTICAL ABSORTION SPECTRA AND COLORIMETRIC DATA OF THE GEMOLOGICAL GARNETS FROM THE SANTO INACIO RIVER BASIN, COROMANDEL COUNTY-MG, BRAZIL The study covered thirty-one samples of gemological garnets of more than $5 \mathrm{~mm}$ in size from the alluvial sediments at the Charneca site and from the Vargem-1 pipe, both located in the Santo Inacio River basin in Coromandel county, State of Minas Gerais. Among these samples, and based on chemical composition data, optica! absorption spectroscopy, and colorimetry, we were able to distinguish three main types of gemological garnets (purplish-red, orangish-red, and yellowish-orange), the hues of which are due to the combination of optically active centers of $\mathrm{Cr}_{\mathrm{IV}}{ }^{5+}, \mathrm{Fe}_{\mathrm{VI}}{ }^{3+}$ and $\mathrm{Fe}_{\mathrm{VIII}}{ }^{2+}$. Comparison of the garnets analyzed with those of the Czech Massif (Linhorka, Czech Republic) revealed that the former feature less saturation of the main color tone, resulting in a more attractive color and enhanced gemological value.

Keywords: garnet, optical absorption spectra, colorimetry, gemology, Coromandel-MG, Brazil

RESUMO Foram estudadas 31 amostras de granadas gemológicas (com dimensões superiores a $5 \mathrm{~mm}$ ) dos sedimentos aluviais da localidade de Charneca e do Pipe Vargem-1, ambos situados na bacia do rio Santo Inácio, no município de Coromandel, Estado de Minas Gerais. Com base nos dados do quimismo mineral, espectroscopia óptica de absorção e da colorimetria, foi possível distinguir dentre elas, três tipos principais de granadas gemológicąs (vermelho-púrpura, vermelho-alaranjada e laranja-amarelada), cujos matizes são devidos à combinação de centros opticamente ativos de $\mathrm{Cr}_{\mathrm{VI}}^{3+}, \mathrm{Fe}_{\mathrm{VI}}^{3+}$ e $\mathrm{Fe}_{\mathrm{VIII}}^{2+}$. As granadas estudadas comparativamente às do Maciço Tcheco (Pipe Linhorka, República Tcheca) apresentam menor saturação do matiz principal, fato que lhes confere uma cor mais agradável e um maior valor gemológico.

Palavras-chave: granada, espectro óptico de absorção, colorimetria, gemologia, Coromandel- MG-Brasil
\end{abstract}

INTRODUÇÃO Embora a aplicação das granadas kimberlíticas em gemologia tenha sido sugerida há cerca de duas décadas por Platonov et al.(1976) e Taran et al.(1978), foi somente no último decénio que o seu emprego obteve impulso e aceitação no mercado de jóias, isso graças a um arrojado marketing de promoção e vendas e ao desenvolvimento e implantacão de novos circuitos de beneficiamento nas unidades de extração de diamantes de kimberlitos na Yakutia (República Sakha) - Rússia que prevêem a separação concomitante como subprodutos a granada, o zircão e a olivina.

A extracão de granadas kimberlíticas esteve organizada em vários pipes da África do Sul (De Beers, Kimberley) e, em aluviões associadas aos pipes Premier, Roberts Victor e nas aluviões do rio Vaal (Kornilov \& Solodova 1983).

Não obstante as granadas abarcarem uma boa fatia do mercado de gemas, verifica-se que dentre elas, a participação das kimberlíticas ainda é pequena.

Hoje, dentre as variedades gemológicas dos minerais do grupo das granadas, são muito apreciados o piropo vermelho, vermelho-púrpura e vermelho-alaranjado da Boêmia-República Tcheca (Fiala \& Padera 1977, Schlüter \& Weitschat 1991), a almandina vermelho-violácea, vermelha, rosa e alaranjada (Kornilov \& Solodova 1983), o piropo-almandina marrom-avermelhado (Kornilov \& Solodova 1983, Schmetzer \& Bank 1981), a espessartita amarelo-alaranjada (Kornilov \& Solodova 1983), a espessartita-piropo-grossulária marrom-amarelada (Schmetzer 1982), o piropo-almandina-espessartita-grossulária alaranjado (Bank 1979), o piropo-espessartita vermelho-rosa e rosa (Schmetzer \& Bank 1981, Stockton \& Manson 1986), a grossulária vermelho-alaranjada e marrom "hessonita", grossulária incolor, verdeclara "tsavorita", verde-amarelada, verde "vivo", a grossulária com cromo e ou com vanádio verde-escura (Bank 1979, 1982, Bridges 1974, Kornilov \& Solodova 1983, Muije et al 1979, Paulitsch 1989), a andradita verde-amarelada "demantóide" e a verde-parda "topazolita" (Bank 1978-1979, 1979, Kornilov \& Solodova 1983, Phillips \& Talantsev 1996, Platonov et al. 1984), a uvarovita verde-esmeralda (Kornilov \& Solodova 1983), as granadas cromíferas e vanadiníferas com "efeito alexandrita" representadas por piropo-grossuláriauvarovita, piropo-uvarovita-grossulária-espessartita e piropo (Bank 1978, 1979, 1979, Saul et al. 1978, Schmetzer et al. 1980, Stockton 1982), o "schorl" e a "melanita" preta (Kornilov \& Solodova 1983), a "hidrogrossulária" verde-amarelada e amarelo-esverdeada (Bank 1982).

A maioria dos gemólogos e joalheiros desconhece que algumas variedades de granadas kimberlíticas se assemelham ao piropo vermelho, vermelho-púrpura e vermelho-alaranjado da Boémia, o qual é utilizados desde o século XII em joalheria e serve ainda como padrão de referência para as granadas de coloração vermelha.
Cabe salientar que as granadas kimberlíticas de um único pipe podem por vezes abarcar praticamente toda a faixa de cores do espectro, inclusive as de matizes púrpuras, excetuando-se as azuis.

Outrossim, são encontradas entre as granadas kimberlíticas variedades únicas em termos de matizes e tonalidades que não se acham entre granadas de outros tipos genéticos. Algumas dessas granadas, que são extremamente raras, se, promovidas comercialmente com habilidade, poderiam alcançar altos preços no mercado.

Uma das singularidades das granadas kimberlíticas estudadas é a presenca de grãos de vários matizes e dimensões relativamente grandes (até $10 \mathrm{~mm}$ ), cuja frequência nos coluviões, eluviões e aluviõ̃es adjacentes ao Pipe Vargem-1 (Fig. 1) é significativamente maior do que nos da Yakutia - Rússia de acordo com os dados de Platonov et al.(1976). Isto sugere a possibilidade da exploração concomitante, ou não, de granadas e diamantes na bacia do rio Santo Inácio.

Por outro lado, a montante da localidade de Charneca, além do Pipe Vargem-1, existem pelo menos outras quatro intrusões kimberlíticas, que liberam granadas nos sedimentos da bacia do rio Santo Inácio.

MÉTODOS O presente trabalho é a primeira tentativa de estudo da natureza da cor e dos parâmetros colorimétricos das granadas

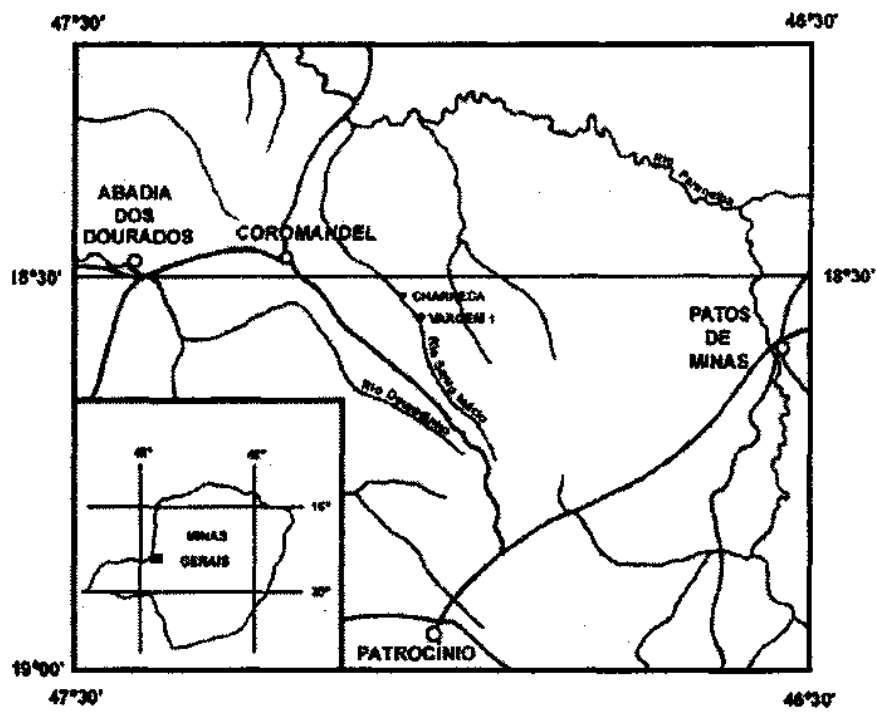

Figura l-Localização do Pipe Vargem-1 e da vila de Charneca, Município de Coromandel, Estado de Minas Gerais.

Universidade Federal de Ouro Preto, Escola de Minas, Departamento de Geologia, CEP 35400, Campus Morro do Cruzeiro s/n, Ouro Preto, MG, Brasil, Endereco atual: Rua Luiz Tolezano N²23, CEP 09400-000, Ribeirão Pires, SP, Brasil

Institute of Geochemistry, Mineralogy and Ore Formation - Ukranian National Academy of Science, 34 Palladina av., Kiev-142, 252680, Ukrania 
kimberlíticas do Brasil, encontradas tanto em fontes primárias, como em coletores secundários, visando seu possível aproveitamento.

Para esse estudo, foram escolhidos em lupa binocular 31 grãos de granadas cobrindo toda a gama de cores e com dimensões superiores a5mm.

$\mathrm{O}$ estudo da composição química das granadas foi realizado em microssonda eletrônica modelo Camebax, no Laboratório de Análises por Microssonda Eletrônica do Instituto de Geologia e Mineralogia da Filial Siberiana da Academia de Ciências da Rússia, na cidade de Novpssibirsk, Rússia, utilizando-se a granada UD-92 como padrão analítico.

A fim de estudar objetivamente as particularidades da natureza da cor (centros opticamente ativos) e os parâmetros colorimétricos das granadas, foram tirados espectros ópticos de absorção

$$
\left(\mathrm{K}=\frac{1}{\mathrm{~d}} \log \frac{\mathrm{YO}}{\mathrm{Y}}, \mathrm{mm}^{-1}\right)
$$

na faixa de 380 a $775 \mathrm{~nm}$. Para tal, foram preparados com as mesmas granadas anteriormente utilizadas na análise por microssonda eletrônica, corpos de prova (após a sua extração das seções polidas) na fornia de lamínulas plano-paralelas especularmente polidas de ambos os lados, com espessura de 0,2 a $0,6 \mathrm{~mm}$.

As medições dos espectros de absorção foram realizadas por meio de um espectrofotômetro construído pela equipe técnica do Instituto de Geoquímica, Mineralogia e Formação de Minérios da Academia Nacional de Ciências da Ucrânia, em Kiev. A descrição do equipamento, os detalhes das técnicas de medição interpretação dos espectros ópticos e os cálculos dos parâmetros colorimétricos: $\mathrm{x}, \mathrm{y}, \mathrm{z}$ (coeficientes de cor); $\lambda_{\mathrm{k}}$ (comprimento de onda do matiz principal no ar); $p_{c}$ (saturação ou pureza do matiz principal); Y (intensidade), podem ser encontrados em Matsyuk, Platonov \& Khomenko (1985). Todos os resultados das medições foram normalizados para lamínulas de $1,0 \mathrm{~mm}$ de espessura.

Para a determinação da paragênese das granadas, com base nos parâmetros colorimétricos, utilizou-se o diagrama colorimétrico $\lambda_{\mathrm{k}}$ $\mathrm{p}_{\mathrm{c}}$ de Matsyuk et al. (1994).

Para a definição das cores das granadas, foi utilizado o diagrama padrão de cromaticidade - triângulo de cromaticidade XYZ da Comissão Internacional de Iluminação -CIE (Gurevitch 1950 e Judd \& Wyszecki 1975).

Objetivando avaliar o potencial gemológico das granadas estudadas, compararam-se os seus dados de parâmetros óptico-espectrométricos (PÕE) e colorimétricos (PC) com os das granadas gemológicas de várias intrusões peridotítico-piroxeníticas e do Pipe Linhorka, da Boémia.

COMPOSIÇÃO QUÍMICA Como se observa nas tabelas 1 e 2 , as granadas estudadas, segundo o seu quimismo, trata-se de piropo com conteúdo variável de moléculas de almandina e uvarovita.

As granadas das aluviões do rio Santo Inácio, na localidade de Charneca, caracterizam-se por apresentar teores pouco variáveis de $\mathrm{CaO}(4,16-4,97 \%), \mathrm{MgO}(19,87-20,67 \%)$ e $\mathrm{FeO}(8,30-9,63 \%)$, bem como teores relativamente baixos de $\mathrm{TiO}_{2}(0,09-0,34 \%)$ (Tab.l). Outrossim, apresentam uma variação um pouco maior nos teores de $\mathrm{Cr}_{2} \mathrm{O}_{3}$ $(1,50-3,89 \%)$ e $\mathrm{Al}_{2} \mathrm{O}_{3}(19,69-21,58 \%)$. Os dados indicam que essas granadas provavelmente representam os produtos finais de um "trend" bem definido de diferenciação de um magma ultrabásico.

O supradito é extensível às granadas do Pipe Vargem-1 (Tab.2), as quais também apresentam variações muito pequenas nos teores dos

Tabela l-Análises químicas das granadas gemológicas da localidade de Charneca (\% em peso).

\begin{tabular}{|c|c|c|c|c|c|c|c|c|c|c|c|}
\hline $\begin{array}{c}\mathrm{N}^{2} \mathrm{da} \\
\text { Amostra }\end{array}$ & $\mathrm{SiO}_{2}$ & $\mathrm{TiO}_{2}$ & $\mathrm{Al}_{2} \mathrm{O}_{3}$ & $\mathrm{C}_{r_{2}} \mathrm{O}_{3}$ & $\mathrm{FeO}$ & $\mathrm{MnO}$ & $\mathrm{MgO}$ & $\mathrm{CaO}$ & $\mathrm{Na}_{2} \mathrm{O}$ & $\mathrm{K}_{2} \mathrm{O}$ & Total \\
\hline$C-2 / 1$ & 40,96 & 0,10 & 20,45 & 2,84 & 8,89 & 0,41 & 19,97 & 4,90 & 0,09 & 0,02 & 98,61 \\
\hline C. $2 / 2$ & 41,38 & 0,23 & 20,33 & 2,56 & 8,69 & 0,45 & 20,02 & 4,83 & 0,04 & 0,00 & 98,53 \\
\hline C $-2 / 3$ & 41,45 & 0,24 & 21,04 & 2,22 & 8,73 & 0,43 & 20,54 & 4,54 & 0,05 & 0,00 & 99,24 \\
\hline C. $-2 / 4$ & 41,42 & 0,32 & 21,26 & 1.60 & 9,47 & 0,44 & 20,25 & 4,45 & 0,07 & 0,01 & 99,29 \\
\hline$C-2 / 5$ & 41,87 & 0,08 & 20,39 & 2,89 & 8,90 & 0,47 & 19,94 & 4,86 & 0,06 & 0,01 & 99,46 \\
\hline C - $2 / 6$ & 40,80 & 0,33 & 20,63 & 1,95 & 9,29 & 0,40 & 20,03 & 4,39 & 0,10 & 0,02 & 97,92 \\
\hline C $-2 / 6$ & 41,30 & 0,33 & 20,74 & 1,84 & 9,22 & 0,44 & 20,09 & 4,40 & 0,07 & 0,02 & 98,42 \\
\hline C - $2 / 6$ & 41,28 & 0,29 & 21,26 & 1,87 & 9,45 & 0,39 & 20,41 & 4,33 & 0,08 & 0,00 & 99,36 \\
\hline $\mathrm{C}-27$ & 41,48 & 0,21 & 19,69 & 3,89 & 8,91 & 0,43 & 19,90 & 4,92 & 0,06 & 0,00 & 99,49 \\
\hline C - $2 / 8$ & 41,54 & 0,31 & 21,52 & 1.79 & 8,94 & 0,44 & 20,67 & 4,52 & 0,09 & 0,01 & 99,81 \\
\hline C $-2 / 9$ & 41,51 & 0,35 & 21,23 & 1,50 & 9,63 & 0,41 & 19,87 & 4,39 & 0,07 & 0,01 & 98,96 \\
\hline $\mathrm{C}-2 / 10$ & 41,85 & 0,22 & 20,12 & 3,52 & 8,59 & 0,42 & 20,29 & 4,97 & 0,03 & 0,00 & 100,00 \\
\hline$C \cdot 2 / 11$ & 41,49 & 0,22 & 19,91 & 3,65 & 8,30 & 0,48 & 20,04 & 4,94 & 0,08 & 0,00 & 99,10 \\
\hline$C-2 / 12$ & 40,95 & 0,23 & 21,37 & 1,63 & 8,96 & 0,39 & 20,38 & 4,21 & 0,06 & 0,00 & 98,18 \\
\hline C. $-2 / 12$ & 41,55 & 0,25 & 21.58 & 1,77 & 9,39 & 0,42 & 20,48 & 4,16 & 0,07 & 0,00 & 99,67 \\
\hline$C \cdot 2 / 13$ & 41,28 & 0,28 & 20,85 & 2,48 & 8,92 & 0,38 & 20,13 & 4,66 & 0,10 & 0,00 & 99,07 \\
\hline$C \cdot 2 / 14$ & 41,59 & 0,20 & 20,63 & 2,50 & 8,70 & 0,42 & 20,24 & 4,69 & 0,03 & 0,00 & 99,00 \\
\hline
\end{tabular}

Tabela 2 - Análises químicas das granadas gemológicas do Pipe Vargem-1(\% em peso).

\begin{tabular}{|cccccccccccc|}
\hline $\begin{array}{c}\mathrm{N}^{\mathrm{i}} \mathrm{da} \\
\text { Amostra }\end{array}$ & $\mathrm{SiO}_{2}$ & $\mathrm{TiO}_{2}$ & $\mathrm{Al}_{2} \mathrm{O}_{3}$ & $\mathrm{Cr}_{2} \mathrm{O}_{3}$ & $\mathrm{FeO}$ & $\mathrm{MnO}$ & $\mathrm{MgO}$ & $\mathrm{CaO}$ & $\mathrm{Na}_{2} \mathrm{O}$ & $\mathrm{K}_{2} \mathrm{O}$ & Total \\
\hline $\mathrm{V}-1 / 1$ & 40,88 & 0,13 & 20,88 & $\mathbf{2 , 4 3}$ & $\mathbf{8 , 9 4}$ & 0,44 & 20,05 & 4,60 & 0,06 & 0,00 & 98,35 \\
$\mathrm{~V}-1 / 2$ & 41,29 & 0,30 & 20,91 & 1,96 & 9,55 & 0,44 & 19,88 & 4,30 & 0,07 & 0,00 & 98,63 \\
$\mathrm{~V}-1 / 3$ & 40,72 & 0,14 & 20,43 & 2,43 & 8,91 & 0,41 & 20,09 & 4,72 & 0,04 & 0,00 & 97,85 \\
$\mathrm{~V}-1 / 3$ & 41,56 & 0,13 & 20,59 & 2,46 & 8,67 & 0,42 & 20,21 & 4,75 & 0,03 & 0,00 & 98,80 \\
$\mathrm{~V}-1 / 4$ & 41,34 & 0,35 & 21,23 & 1,75 & 9,85 & 0,43 & 20,06 & 4,38 & 0,06 & 0,00 & 99,38 \\
$\mathrm{~V}-1 / 5$ & 41,62 & 0,27 & 21,10 & 1,69 & 9,58 & 0,43 & 19,99 & 4,23 & 0,09 & 0,00 & 98,91 \\
$\mathrm{~V}-1 / 6$ & 41,17 & 0,09 & 20,54 & 2,73 & 9,12 & 0,49 & 19,81 & 4,79 & 0,04 & 0,00 & 98,74 \\
$\mathrm{~V}-1 / 7$ & 41,15 & 0,17 & 20,76 & 2,15 & 9,27 & 0,39 & 19,95 & 4,38 & 0,05 & 0,00 & 98,21 \\
$\mathrm{~V}-1 / 7$ & 41,69 & 0,13 & 20,80 & 2,31 & 9,20 & 0,47 & 20,12 & 4,54 & 0,04 & 0,00 & 99,26 \\
$\mathrm{~V}-1 / 8$ & 41,52 & 0,26 & 21,27 & 1,47 & 9,51 & 0,42 & 20,08 & 4,17 & 0,09 & 0,00 & 98,70 \\
$\mathrm{~V}-1 / 9$ & 41,29 & 0,27 & 21,34 & 1,43 & 9,42 & 0,43 & 20,39 & 4,24 & 0,05 & 0,01 & 98,80 \\
$\mathrm{~V}-1 / 10$ & 41,49 & 0,34 & 21,28 & 1,64 & 9,72 & 0,42 & 19,84 & 4,34 & 0,05 & 0,00 & 99,07 \\
$\mathrm{~V}-1 / 11$ & 41,33 & 0,08 & 19,81 & 3,60 & 8,80 & 0,53 & 19,82 & 5,34 & 0,04 & 0,01 & 99,31 \\
$\mathrm{~V}-1 / 12$ & 41,08 & 0,18 & 18,66 & 5,03 & 8,62 & 0,44 & 19,66 & 5,22 & 0,07 & 0,03 & 98,89 \\
$\mathrm{~V}-1 / 13$ & 41,22 & 0,28 & 20,87 & 1,83 & 8,97 & 0,42 & 20,22 & 4,65 & 0,06 & 0,00 & 98,46 \\
$\mathrm{~V}-1 / 14$ & 40,97 & 0,28 & 20,39 & 2,59 & 8,92 & 0,44 & 20,15 & 4,71 & 0,06 & 0,01 & 98,45 \\
$\mathrm{~V}-1 / 15$ & 41,50 & 0,09 & 20,74 & 2,73 & 8,67 & 0,46 & 20,32 & 4,76 & 0,06 & 0,01 & 99,26 \\
$\mathrm{~V}-1 / 16$ & 41,57 & 0,30 & 21,40 & 1,41 & 9,46 & 0,36 & 19,97 & 4,24 & 0,07 & 0,01 & 98,71 \\
$\mathrm{~V}-1 / 17$ & 41,71 & 0,25 & 21,30 & 1,48 & 9,55 & 0,45 & 20,19 & 4,18 & 0,07 & 0,01 & 99,11 \\
\hline
\end{tabular}


seus óxidos constituintes. São poucas as granadas do pipe que apresentam teores médios de $\mathrm{Cr}_{2} \mathrm{O}_{3}$ (no máximo de 5,03\%) e $\mathrm{CaO}$ (no máximo de $5,22 \%$ ).

No todo, quanto à composição química, as amostras estudadas assemelham-se às granadas de paragêneses Iherzolíticas e websteríticas encontradas em kimberlitos. No entanto chamam a atenção pelo seu alto teor em $\mathrm{FeO}$ (que atinge 9,85\%), que as diferencia, por exemplo, das granadas dessas paragêneses encontradas nos kimberlitos da Yakutia - Rússia, nas quais o teor de $\mathrm{FeO}$ raramente ultrapassa os $8,00 \%$, não obstante o teor análogo de $\mathrm{Cr}_{2} \mathrm{O}_{3}, \mathrm{MgO}$ e $\mathrm{CaO}$. Não se pode excluir que este fato esteja relacionado com a composição específica do meio de cristalizacão à partir do qual esses minerais mantélicos se formaram. Esta dúvida poderá ser resolvida com 0 estudo complementar das granadas de outras frações granulométricas.

ESPECTROS ÓPTICOS E COLORAÇÃO Com base nos espectros ópticos de absorção típicos (Figs. 2 e 3 ) e nos parâmetros colorimétricos (Tab. 3 e 4), pode-se subdividir as granadas em três grupos de cor: vermelho-púrpura $\left(\lambda \mathrm{k}=500-630 \mathrm{~nm}, \mathrm{p}_{\mathrm{c}}=0,250-0,550\right.$ unid. rel.), vermelho-alaranjadas $\left(\lambda \mathrm{k}=630-590 \mathrm{~nm}, \mathrm{p}_{\mathrm{c}}=0,300-0,700\right.$ unid. rel.) e laranja-amareladas $\left(\lambda \mathrm{k}=590-584 \mathrm{~nm}, \mathrm{p}_{\mathrm{c}}=0,500-0,750\right.$ unid. rel.). Cabe ressaltar que esta subdivisão é formal, pois na verdade existem transições gradativas entre as três cores e que cada uma das populações estudadas exibem suítes específicas de cores com baixa intensidade de saturação do matiz principal (Tab. 3 e 4, Fig. 3).

Nas figuras 2 e 3, tem-se, respectivamente, os espectros ópticos de absorção característicos para as variedades de cor de granadas encontradas em Charneca e no Pipe Vargem-1. Nelas vemos que a cor das

Tabela 3 - Parâmetros colorimétricos e paragênese das granadas gemológicas da localidade de Charneca.

\begin{tabular}{|c|c|c|c|c|c|c|}
\hline \multirow{2}{*}{$\begin{array}{c}N^{\circ} \mathrm{DA} \\
\text { AMOSTRA }\end{array}$} & \multicolumn{5}{|c|}{$\begin{array}{l}\text { PARAMETROS COLORIMÉTRICOS } \\
\text { (para plaquinha com } 1 \mathrm{~mm} \text { de espessura) }\end{array}$} & \multirow[t]{2}{*}{ PARAGÉNESE } \\
\hline & $x$ & $y$ & $\lambda_{4,} \mathrm{~nm}$ & $\begin{array}{l}\text { Pos unid. } \\
\text { rel. }\end{array}$ & $Y, \%$ & \\
\hline$c-2 / 1$ & 0,456 & 0,320 & 612,9 & 0,400 & 12,2 & L (GLE) \\
\hline$C \cdot 2 / 2$ & 0,479 & 0,331 & 606,5 & $0, \overline{492}$ & 13,3 & L.GLE) \\
\hline$C \cdot 2 / 3$ & 0,469 & 0,342 & 601,0 & $0, \overline{494}$ & 17.1 & L(GLE) \\
\hline$C-2 / 4$ & 0,492 & 0,363 & 595,7 & 0,614 & 16,4 & $L$ (LPC) \\
\hline$C=2 / 5$ & 0,460 & 0,311 & 622,2 & 0,388 & 12,6 & L (GLE) \\
\hline$C-2 / 6$ & 0,510 & 0,360 & 597,6 & 0,655 & 15,2 & $\mathrm{~L}(\mathrm{LPC})$ \\
\hline$C-2 / 7$ & 0,513 & $0,3 \pm 1$ & 621,3 & 0,528 & 7,6 & L (LPC) \\
\hline $\mathrm{C} \cdot \overline{2 / 8}$ & 0,466 & 0,367 & 593,0 & 0,555 & 23,1 & $\mathrm{~L}(\mathrm{GLE})$ \\
\hline C $-2 / 9$ & 0,478 & 0,374 & 592,3 & 0,605 & 21,2 & L (GLE) \\
\hline$C \cdot 2 / 10$ & 0,456 & 0,317 & 615,6 & 0,393 & 11,2 & L (GLE) \\
\hline$C \cdot 2 / 11$ & 0,447 & 0,357 & 594,2 & 0,475 & 24,0 & L (GLE) \\
\hline$C \cdot 2 / 12$ & 0,446 & 0,343 & 598,9 & 0,435 & 19,9 & L (GLE) \\
\hline$c \cdot 2 / 13$ & 0,447 & 0,335 & 602,5 & 0,417 & 17,7 & $L(G L)$ \\
\hline
\end{tabular}

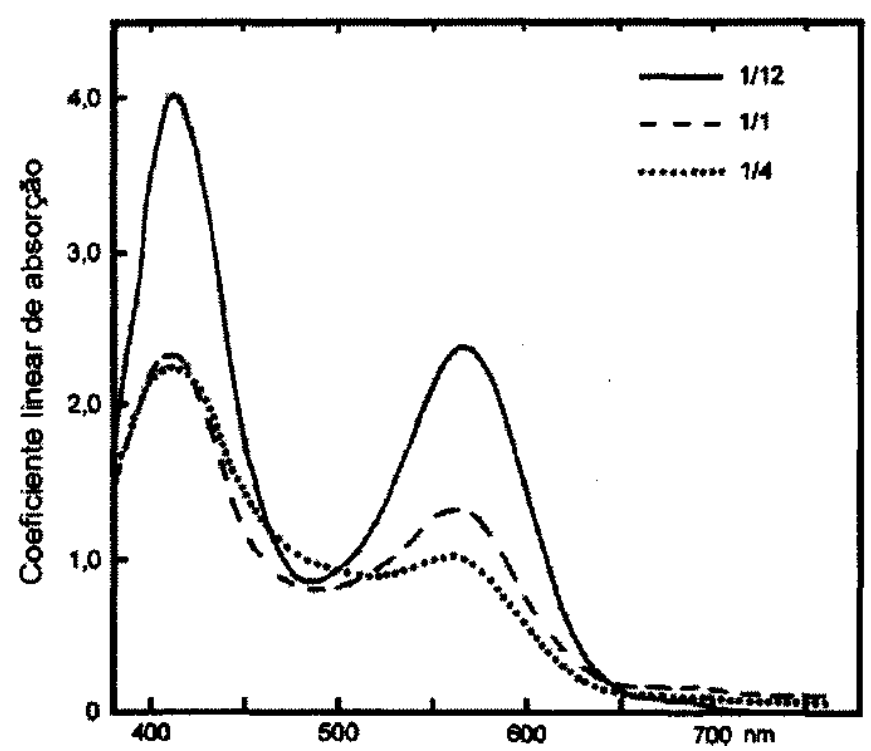

Figura 2 - Espectros ópticos de absorção típicos das granadas gemológicas do Pipe Vargem-1 granadas deve-se principalmente aos íons $\mathrm{Cr}^{3+} \mathrm{e} \mathrm{Fe}^{2+}$. Os íons $\mathrm{Fe}^{3+}$ exercem menor influência na coloração das granadas, os quais relacionam-se à banda de transferência de carga $\mathrm{O}^{2-} \longrightarrow \mathrm{Fe}^{3+}$.

Nos espectros ópticos de granadas de cor vermelho-púrpura (Fig. 2, amostra 1/12; Fig. 3, amostra 2/6) vêm-se as bandas de absorção relativamente intensas, relacionadas com os centros opticamente ativos $(\mathrm{COA})$ de $\mathrm{Cr}_{\mathrm{VI}}{ }^{3+}\left(\mathrm{Eu}=17.600-17.700 \mathrm{~cm}^{-1}, \mathrm{E}_{\mathrm{Y}}=24.000-\right.$ $24.200 \mathrm{~cm}^{-1}$ ), bem como uma banda de absorção fraca na região dos $505 \mathrm{~nm}$, atribuída às transições proibidas $\mathrm{d}$-d nos íons $\mathrm{Fe}_{\mathrm{VIII}}$

Por outro lado, nos espectros das granadas vermelho-alaranjadas (Fig. 2, amostra 1/1; Fig. 3, amostra 2/6) e sobretudo nas laranjaamareladas (Fig. 2, amostra 1/1; Fig. 3 amostra 2/11), ocorre um decréscimo na intensidade das bandas $U$ de absorção dos COA de, $\mathrm{Cr}_{\mathrm{VI}}{ }^{3+}$ havendo com isso, uma diminuição da influência desses COA na composição da cor da granada.

Simultaneamente -ocorre 0 aumento da função dos íons $\mathrm{Fe}^{2+} \mathrm{e}$ sobretudo dos íons $\mathrm{Fe}^{3+}$ no desenvolvimento da coloração amarela.

A análise das características colorimétricas das granadas gemológicas das aluviões da localidade de Charneca e do Pipe Vargem-1 mostram que elas apresentam aproximadamente a mesma faixa de

Tabela 4 - Parâmetros colorimétricos e paragênese das granadas gemológicas do Pipe Vargem-1.

\begin{tabular}{|c|c|c|c|c|c|c|}
\hline \multirow{2}{*}{$\begin{array}{c}\text { No DA } \\
\text { AMOSTRA }\end{array}$} & \multicolumn{5}{|c|}{$\begin{array}{l}\text { PARÀMETROS COLORIMETRICOS } \\
\text { (para plaquinha com } 1 \mathrm{~mm} \text { de espessura) }\end{array}$} & \multirow[t]{2}{*}{ PARAGENESE } \\
\hline & $x$ & $\mathbf{y}$ & $\lambda_{k,} \mathrm{~nm}$ & $\begin{array}{l}\mathrm{p}_{\mathrm{c}} \text {, unid. } \\
\text { rel. }\end{array}$ & $\mathbf{Y}, \%$ & \\
\hline $\mathrm{V}-\mathrm{b} / 1$ & 0,455 & 0,325 & 609,2 & 0,411 & 13,5 & L (GLE) \\
\hline$v-1 / 2$ & 0,508 & 0,363 & 596,8 & 0,655 & 16,2 & L (LPC) \\
\hline $\mathrm{V}-1 / 3$ & 0,459 & 0,324 & 610,0 & 0,420 & 17,0 & L (GLE) \\
\hline$V-1 / 4$ & 0,509 & 0,371 & 594,9 & 0,679 & 18,1 & L (LPC) \\
\hline$V \cdot 1 / 5$ & 0,472 & 0,357 & 596,1 & 0,544 & 183 & L (GLE) \\
\hline$V-1 / 6$ & 0,484 & 0,355 & 597,4 & 0,572 & 20,8 & L (GLE) \\
\hline$v+1 / 7$ & 0,444 & 0,322 & 610,5 & 0,376 & 16,8 & L (GLE) \\
\hline$V-1 / 8$ & 0,491 & 0,367 & 594,9 & 0,621 & 17,1 & L (LPC) \\
\hline$V=1 / 9$ & 0,479 & 0,370 & 593,3 & 0,597 & 27,8 & L (GLE) \\
\hline $\mathrm{V}-1 / 10$ & 0,513 & 0,372 & 595,1 & 0,693 & 19,1 & L (LPC) \\
\hline $\bar{v}-1 / 11$ & 0,453 & 0,296 & 492,6 & 0,346 & 9,5 & L (SGEE) \\
\hline$v-1 / 12$ & 0,484 & 0,290 & 492,7 & 0,430 & 7,0 & Wh (GWH) \\
\hline$V=1 / 13$ & 0,480 & 0,332 & 605,6 & 0,498 & 17,7 & L (GLE) \\
\hline$V-1 / 14$ & 0,488 & 0,342 & 602,0 & 0,547 & 13,5 & L (GLE) \\
\hline$V-1 / 15$ & 0,445 & 0,312 & 622,4 & 0,350 & 17,1 & L(GLE) \\
\hline$V=1 / 16$ & 0,472 & 0,317 & 615,3 & 0,438 & 14,4 & L (GLE) \\
\hline $\mathrm{V}-1 / 17$ & 0,482 & 0,367 & 594,1 & 0.597 & 23,8 & L (GLE) \\
\hline
\end{tabular}

$\mathrm{L}$ - paragênese Iherzolítica ( $\mathrm{gr}+\mathrm{ol}+\mathrm{cpx}+\mathrm{opx} \pm \mathrm{spl})$, GLE - granada lherzolito equigrânular isótropo;

SGLE- espinélio- granada lherzolito equigrânular isótropo;

LPC - lherzolito porfirítico cisalhado;

Wh - paragênese wehrlítica [ $\mathrm{gr}+\mathrm{ol}+\mathrm{cpx} \pm \mathrm{opx}(<3 \%)$; Gwh - granada wehrlito (com granada de cor vermelho-púrpura).

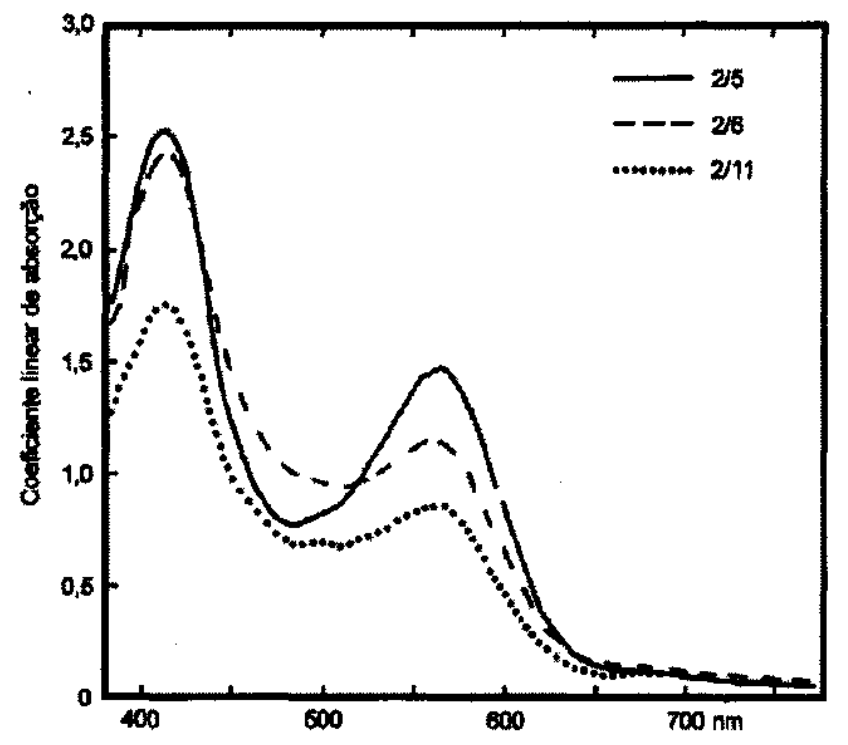

Figura 3 - Espectros ópticos de absorção típicos das granadas gemológicas dos aluviões da localidade de Charneca. 


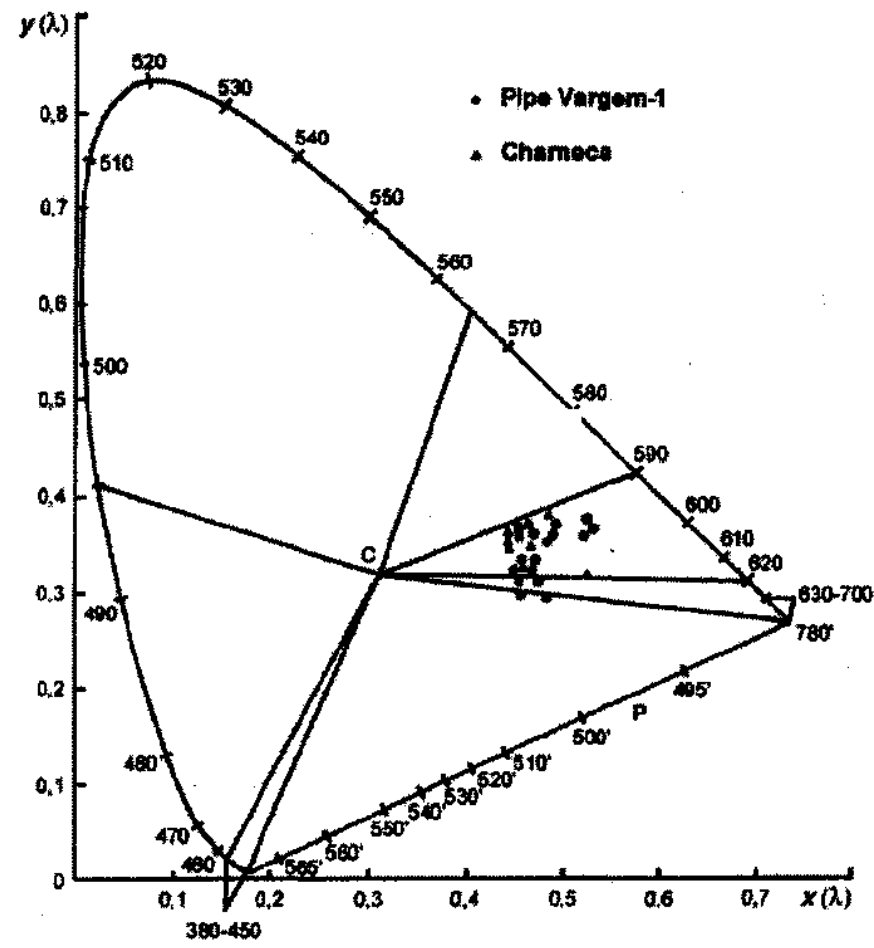

Figura 4 - Diagrama tricromático no sistema XYZ da CIE das granadas gemológicas do Pipe Vargem-1. cores, com predomínio das variedades de matiz vermelho sobre os outros (Fig. 4).

PARAGÊNESE DAS GRANADAS Conforme mencionado, a discriminação paragenética das granadas foi feita com o concurso do diagrama colorimétrico $\lambda \mathrm{k}-\mathrm{P}_{\mathrm{C}}$ de Matsyuk et al. (1994) (Fig. 5). Na figura 6 vê-se que a maior parte das granadas estudadas, tanto as de Charneca, como as do Pipe Vargem-1, são predominantemente de paragênese lherzolítica (granada Iherzolitos equigranulares isótropos, espinélio-granada Iherzolitos equigranulares isótropos) e subordinadamente de paragênese wehrlítica (granada wehrlitos). É possível que algumas das granadas possam ser produto da desintegracão de dunitos e Iherzolitos porfiríticos cisalhados. Essa é uma questão aberta, que poderá ser resolvida com estudos complementares de granadas (com população estatisticamente representativa) da fração mais fina $(0,5-$ $1.0 \mathrm{~mm})$.

DISCUSSÃO DOS RESULTADOS O piropo vermelho, vermelho-púrpura e vermelho-alaranjado (3mm) da Boémia (Fiala 1965 , Faiala \& Padera 1977, Schlüter \& Weitschat 1991) durante muito tempo serviu de padrão como granada para joalheria.

Para fins de comparação com as granadas estudadas, tem-se, respectivamente, nas figuras 7 e 8 , o diagrama de cromaticidade no sistema XYZ (CIE) e o diagrama colorimétrico dos parâmetros $\lambda_{\mathrm{k}}-\mathrm{p}_{\mathrm{c}}$ das granadas da Boémia, extraídos do trabalho de Matsyuk et al. (1987).

As figuras 6 e 8 mostram que o piropo da Boémia e o da bacia do rio Santo Inácio apresentam o mesmo matiz principal de cor. Como distinção, o piropo da Boémia, exibe maior saturação do matiz principal $\left[\mathrm{p}_{\mathrm{c}}=0,400-0,600\right.$ unid. rel. $\left(\lambda_{\mathrm{k}}=492,5 \mathrm{~nm}\right), \mathrm{p}_{\mathrm{c}}=0,600-0,950$ unid rel. $\left.\left(\lambda_{\mathrm{k}}=600 \mathrm{~nm}\right)\right]$. Este fato está relacionado a um maior conteúdo de íons cromóforos $\mathrm{Fe}^{3+}$. Com pouca iluminação diurna ou noturna, $\mathrm{o}$ piropo da Boémia, montado em peças de joalheria, apresenta-se mais

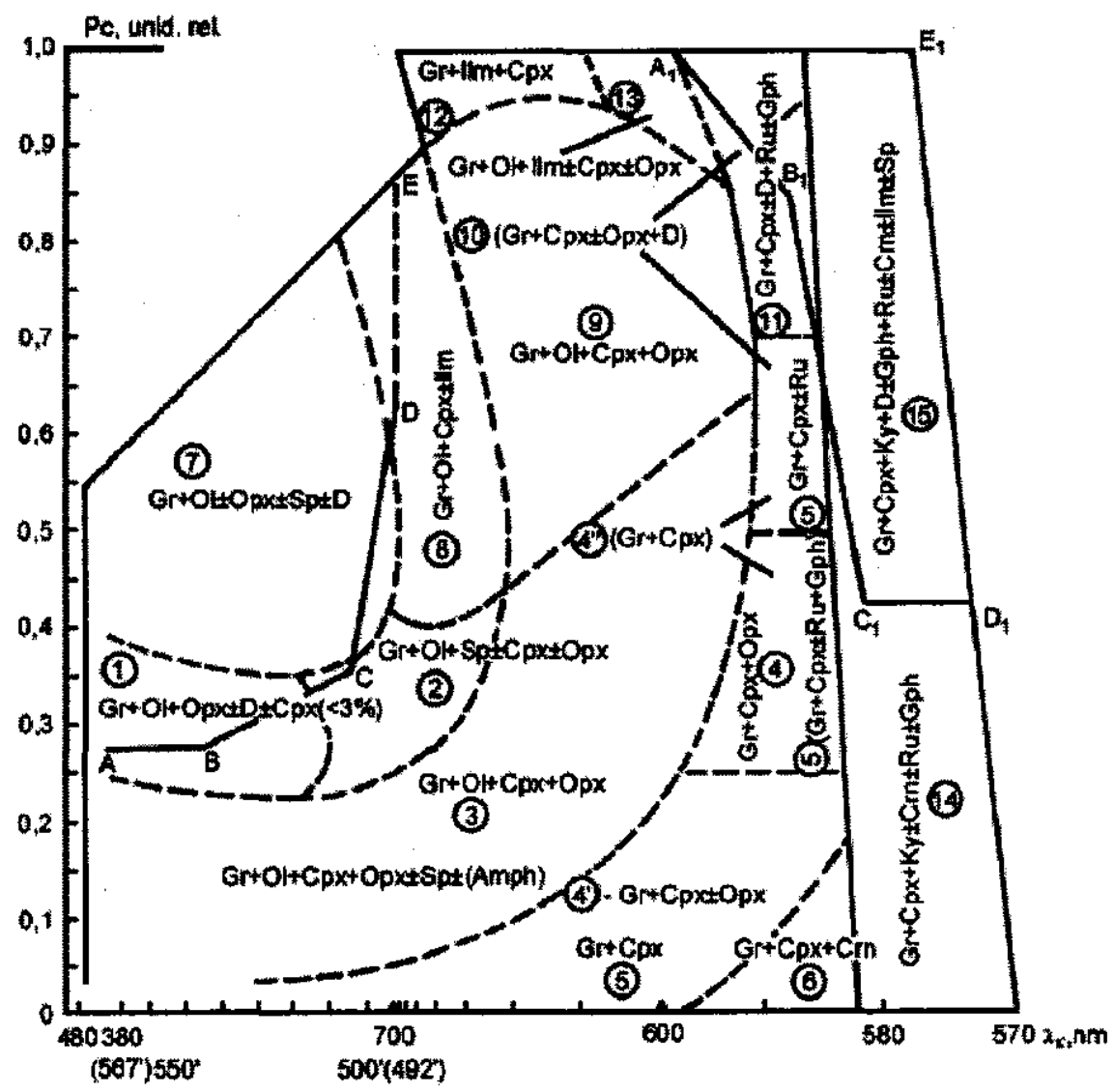

Figura 5 - Diagrama colorimétrico das granadas de paragênese mantélica encontradas em kimberlitos (Matsyuk 1992). 1-15 campos dos matizes das granadas de: ABCDE e AIBICIDIEI - limites dos campos de cor das granadas de paragênese diamantífera de composição ultrabásica e básica respectivamente. 1-Harzburgitos-II; 2- Dunitos e peridotitos com espinélio; 3- Lherzolitos holocristalinos; 4- Mg-websteritos; 4'Mg-clinopiroxenitos; 5- Mg-eclogitos; 6- Coríndon eclogitos; 7- Dunitos, harzburgitos-1; 8-Wehrlitos com granada vermelho-púrpura; 9Iherzolitos cisalhados; 10- Ca-Mg-websteritos e clinopiroxenitos; 11- Mg-Fe eclogitos; 12- Ilmenita-granada clinopiroxênio (intercrescimentos); 13- Maioria dos ilmenita peridotitos; 14- Distênio eclogitos e grospiditos; 15-Distênio, coríndon e coesita eclogitos e grospiditos. 


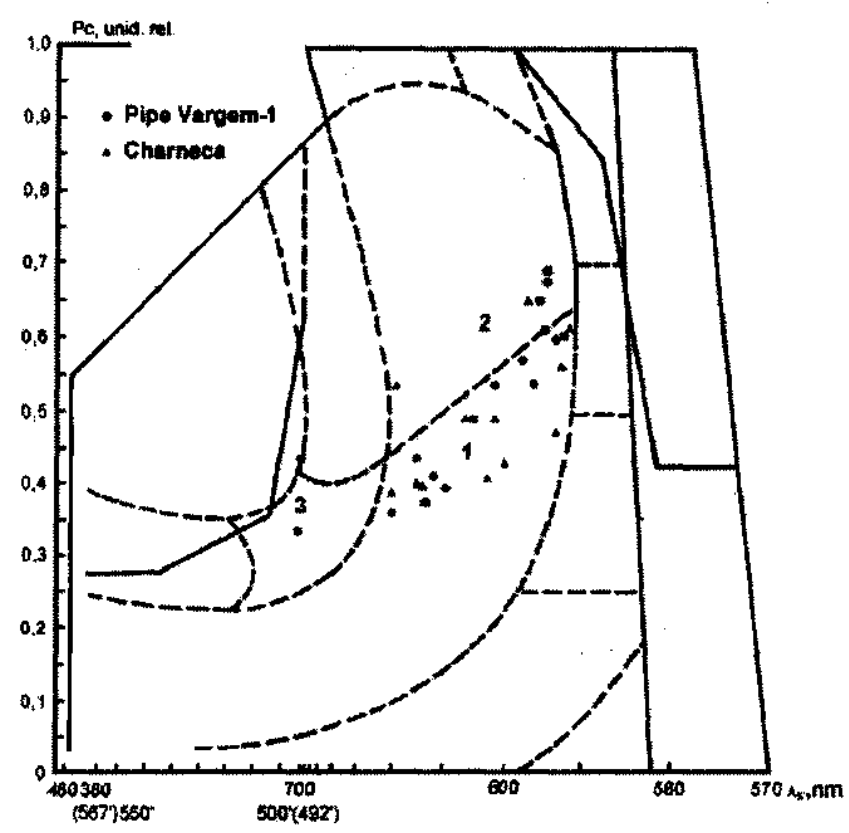

Figura 6 - Diagrama colorimétríco $k$ versus $p_{c}$ das granadas gemológicas do Pipe Vargem-1 e das aluviões da vila de Charneca, l- Lherzólitos equigranulares; 2-Lherzólitos porfirídicos (cisaIhados); 3-Wehrlitos.

escuro e além disso, alguns cristais chegam a possuir tons acinzentados, fato que os deprecia no mercado (Smit 1980).

A maior parte do piropo estudado (cerca de $90 \%$ ) possui grau de saturacão do matiz principal menor que o da Boémia $\left(\mathrm{p}_{\mathrm{c}} 0,600\right.$ unid. rel.)- Esse fato mostra que as granadas lapidadas e montadas em jóias parecerão mais claras que as da Boémia, mesmo sob iluminação fraca.

CONCLUSÕES Os dados do estudo do quimismo mineral, espectroscopia óptica de absorção e colorimetria das granadas gemológicas da localidade de Charneca e do Pipe Vargem-1, permitem tirar as seguintes conclusões:

1 - dentre as granadas gemológicas de dimensões entre 5 e 1 Omm, foram identificados piropo de cor vermelho-púrpura, vermelho-violácea, vermelha, vermelho-alaranjada, alaranjada, laranja-amarelada cujas colorações devem-se a diferentes proporções e relações dos COA causadas por $\mathrm{Crvi}^{3+}, \mathrm{Fevi}^{3+}, \mathrm{Fe}_{\mathrm{VIII}}$

2- nos sedimentos aluviais da localidade de Charneca, tem-se, em ordem decrescente de frequência, granadas com as seguintes cores: laranja-amarelada, laranja, laranja-avermelhada, vermelho-alaranjada, vermelha e vermelho-violácea (vermelho-púrpura).

3- no Pipe Vargem-1 tem-se, em ordem decrescente de frequência, granadas com as seguintes cores: laranja, laranja-avermelhada, vermelha, vermelho-violácea e púrpura.

4- em ambas as localidades, as granadas possuem menor saturação do matiz principal que as de Linhorka (Boémia), isto é, possuem cores mais claras e, consequentemente, são mais adequadas para a confeccão de peças lapidadas de maior tamanho que as da República Tcheca, tidas como padrão de referência de granadas gemológicas de cores vermelho-alaranjadas, vermelhas e vermelho-púrpuras.

5- em vista do exposto, recomenda-se a realização de um estudo sistemático de depósitos similares para caracterizar colorimetricamente as granadas gemológicas e posterior avaliação do seu potencial económico, visando a sua extração concomitante, ou não, com o diamante.

Agradecimentos Um dos autores (W.I.) agradece à Fundação de Amparo à Pesquisa do Estado de Minas Gerais (FAPEMIG) pela concessão da Bolsa de Récem-Doutor, processo CEX 97.995 junto ao DEGEO-EM-UFOP. Ao Prof Antônio Gomes de Araújo, ex-Diretor da Escola de Minas (UFOP) e ao Prof. Dr. Paulo César de Souza, ex-Chefe do Departamento de Geologia-EM-UFOP, pelo incentivo

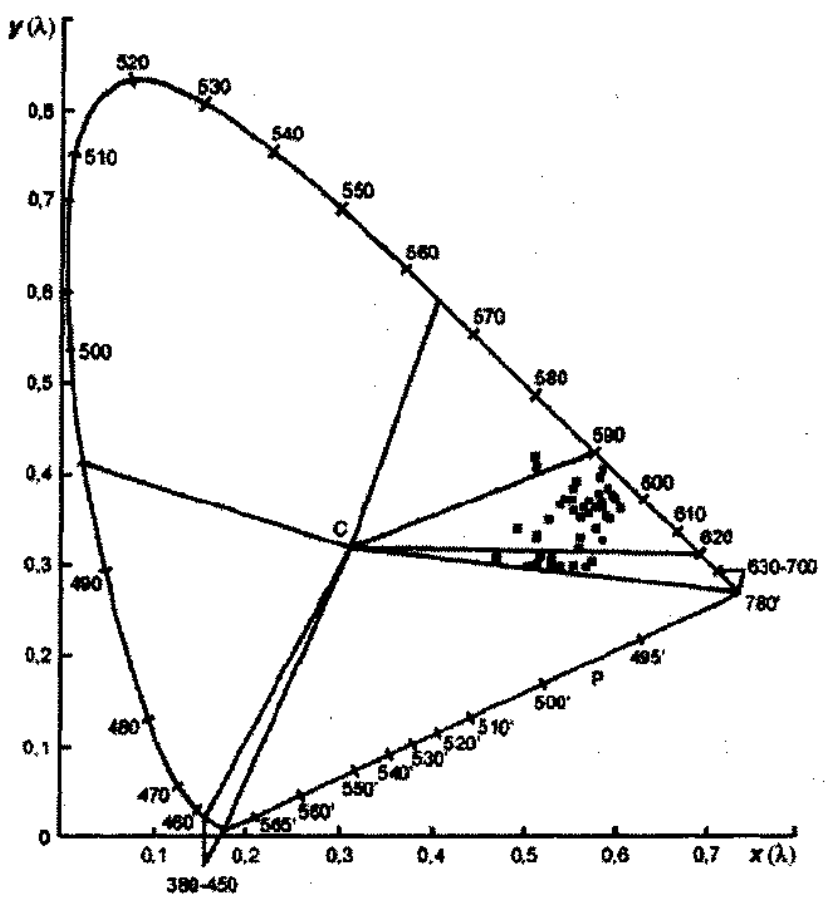

Figura 7 - Diagrama tricromático no sistema XYZ da CIE das granadas gemológicas amostradas em sedimentos aluviais e nas intrusões peridotitíco- piroxeníticas na região do Pipe Linhorka, República Tcheca.

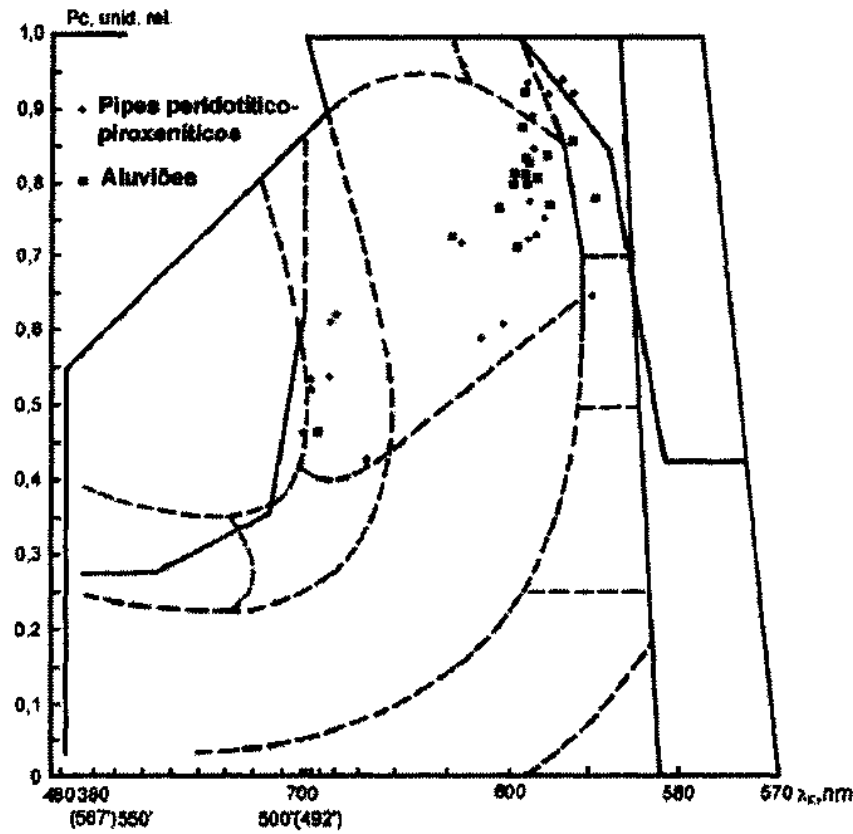

Figura 8 - Diagrama colorimétrico $k$ versus $p_{c}$ das granadas gemológicas amostradas em sedimentos aluviais e nas intrusões peridotíticopiroxeníticas na região do Pipe Linhorka, República Tcheca.

para efetuar estágios técnico-científicos no Institute of Geochemistry, Mineralogy and Ore Formation - Ukranian National Academy of Sciences, Kiev- Ucrânia e no Instituto de Geologia e Mineralogia, da Filial Siberiana da Academia de Ciências da Rússia em NovossibirskRússia, e a dois revisores anónimos que permitiram melhorar o texto do artigo. 


\section{Referências}

Bank H. 1978-1979. Gemological notes. Gems \& Gemol.,16:123-125.

Bank H. 1979. Orange ("Padparadscha") und andersfarbige granate aus Ostafrika. Z. Dtsch GemmoLGes., 28: 146-147.

Bank H. 1982. Über grossular und hydrogrossular. Z. Dtsch. Gemmol. Gês., 31: 93-96.

Bank H. Schnetzer K., Probst S. 1979. Vanadiumgrossulare mit hohe lichtbrechung. Z. Dtsch. Gemmol. G«.,28: 70-72.

Bridges C.R.1974. Green grossular garnets ("tsavorites") in East África. Gems \& Gemol., 14:290-295.

Gurevich M.M. 1950. A cor e a sua medição. Leningrado, Editora Ac.Ciênc.URSS, 266p.

Fiala J. 1965. Pyrope of some garnet-peridotites of the Czech Massif. Krystalinikum, 3:55-74.

Fiala J. \& Padera K. 1977. The chemistry of minerais of the pyrope dunite from borehole T-7 near Staré (Bohemia). Tschermaks Mineral. Petrogr. Mitt. 24:205-219.

Judd D.B. \& Wyszeki G. 1975. Color in Business, Science and Industry. New York, John Wiley \& Sons Inc., $553 \mathrm{p}$

Kornilov N.I. \& Solodova Yu.P. 1983. Pedras Preciosas. Moscou, Nedra, 239 p. (em russo).

Matsyuk S.S. 1992 Cristaloquímica e Tipomorfismo de Minerais de Rochas Mantélicas Segundo Dados de Espectroscopia Óptica). Instituto de Geoquímica e Física de Minerais. Academia de Ciências da Ucrânia, Kiev, Tese de Livre-Docência, Resumo, 38p. (em russo).

Matsyuk S.S., Ilupin I.P., Smirnov G.I., Cherenkov A.F. 1994. Estudo óptico-espectroscópico de megacristais de granadas de kimberlitos da Yakutia e Africa. Mineral. Zhurnal, 16:62-74 (em russo)

Matsyuk S.S., Platonov A.N., Khomenko V.M. 1985. Espectros Ópticos e a Coloração dos Minerais Mantélicos de Kimberlitos. Kiev, Naukova Dumka, 246 p. (em russo).

Matsyuk S.S., Platonov A.N., Vishnevskiy A.A., Kharkiv A.D. 1987. Particularidades da composição química e características óptico-espectroscópicas das granadas de intrusõ es peridotítico-piroxeníticas do Maciço Tcheco. Mineral. Zhurnal, 9:15-28 (em russo).

Muije P., Muije C.S., Muije L.E. 1979. Colorless and green grossularite from Tanzania. Gems \& Gemol.,16:162-173.

Paulitsch P. 1989. Eine mineralogische reise nach Kenia. Aufschluss, 40:383-390.
Phillips Wm.R.\&TalantsevA.S. 1996. Russian demantoid, czar of the garnet family. Gemi \& Gemol., 32:100-111.

Platonov A.N., Taran M.N., Kharkiv A.D., Polshin E. V., Botkunov A.1.1976. Coloração das granadas gemoló gicas de pipes kimberlíticos da Yakutia. Constituição e Propriedades dos Minerais. 12:88-103.

Platonov A.N., Taran M.N., Balitskiy V.S. 1984. A Natureza da Coloração das Gemas. Moscou, Nedra, 196 p. (em russo).

Saul J.M., Jobbins E.A., Statham P.M., Young B.R. 1978. Un essai d'établissement d'une échelle de couleurs pour des grenats de Ia vallée de 1'Umba, Tanzanie. Ver. Gemmol. A.f.g., 54:9.

Schlüter J. \& Weitschat W. 1991. Bohemian garnet today. Gems \& Gemol., 27:168-173. Schmetzer K. 1982. Ein ungewõhnlicher granai aus dem Umba-Tal, Tansania. Z. Dtsch. Gemmol. Gês., 31:59-64.

Schmetzer K. \& Bank H. 1981. Garnets from Umba-Tal valley, Tanzania - members of the solid solution series pyrope-spessartine. NeuesJahrb. Miner. Monatsh., 8:349-354.

Schmetzer K., Bank H., Gubelin E. 1980. The alexandrite effect in minerais: chrysoberyl, garnet, corundum, fluorite. Neues Jahrb. Mineral. Abh., 138:147-164

Smit G. 1980. Pedras Preciosas. Moscou, Mir, 586 p. (tradução russa do original em inglês).

Stockton C.M. 1982. Two notable color-change garnets. Gems \& Gemol, 18:100-101.

Stockton C.M. \& Manson D.V. 1986. The chemical and spectral characteristics of gem garnets from East Africa $l 4^{\text {th }}$ Gen. Meet. Int. Miner. Assoe., Stanford.Calif., 13-18 July, Abstr. Program., Washington D.C., p.239.

Taran M.N., Platonov A.N., Polshin E.V.1978. A coloração das granadas de jazidas da URSS. Constituição e Propriedades dos Minerais, 12: 88-103 (em russo).

Manuscrito A-1038

Recebido em 06 de novembro de 1998 Revisão dos autores em 15 de setembro de 1999 Revisão aceita em 25 de setembro de 1999 